\title{
STAT3 accelerates uterine epithelial regeneration in a mouse model of decellularized uterine matrix transplantation
}

Takehiro Hiraoka, ${ }^{1}$ Yasushi Hirota,,${ }^{1,2}$ Tomoko Saito-Fujita, ${ }^{1}$ Mitsunori Matsuo, ${ }^{1}$ Mahiro Egashira, ${ }^{1}$ Leona Matsumoto, ${ }^{1}$ Hirofumi Haraguchi, ${ }^{1}$ Sudhansu K. Dey, ${ }^{3}$ Katsuko S. Furukawa, ${ }^{4}$ Tomoyuki Fujii, ${ }^{1}$ and Yutaka Osuga'

'Department of Obstetrics and Gynecology, Graduate School of Medicine, The University of Tokyo, Tokyo, Japan.

${ }^{2}$ Precursory Research for Innovative Medical Care (PRIME), Japan Agency for Medical Research and Development (AMED),

Tokyo, Japan. ${ }^{3}$ Division of Reproductive Sciences, Cincinnati Children's Hospital Medical Center, Cincinnati, Ohio, USA.

${ }^{4}$ Department of Bioengineering, School of Engineering, The University of Tokyo, Tokyo, Japan.

\begin{abstract}
Although a close connection between uterine regeneration and successful pregnancy in both humans and mice has been consistently observed, its molecular basis remains unclear. We here established a mouse model of decellularized uterine matrix (DUM) transplantation. Resected mouse uteri were processed with SDS to make DUMs without any intact cells. DUMs were transplanted into the mouse uteri with artificially induced defects, and all the uterine layers were recovered at the DUM transplantation sites within a month. In the regenerated uteri, normal hormone responsiveness in early pregnancy was observed, suggesting the regeneration of functional uteri. Uterine epithelial cells rapidly migrated and formed a normal uterine epithelial layer within a week, indicating a robust epithelial-regenerating capacity. Stromal and myometrial regeneration occurred following epithelial regeneration. In ovariectomized mice, uterine regeneration of the DUM transplantation was similarly observed, suggesting that ovarian hormones are not essential for this regeneration process. Importantly, the regenerating epithelium around the DUM demonstrated heightened STAT3 phosphorylation and cell proliferation, which was suppressed in uteri of Stat3 conditional knockout mice. These data suggest a key role of STAT3 in the initial step of the uterine regeneration process. The DUM transplantation model is a powerful tool for uterine regeneration research.
\end{abstract}

Authorship note: T. Hiraoka, Y. Hirota, and T. Saito-Fujita contributed equally to this work.

Conflict of interest: The authors have declared that no conflict of interest exists.

Submitted: March 15, 2016 Accepted: April 28, 2016 Published: June 2, 2016

Reference information: JCl Insight. 2016;1(8):e87591. doi:10.1172/jici.nsight.87591.

\section{Introduction}

The human uterus exhibits cyclic endometrial renewal every menstrual cycle to prepare for pregnancy. The mouse uterus also shows rapid reconstruction after parturition to prepare for next pregnancy. These findings underscore the high potential of uterine regeneration supporting successful pregnancy in humans and mice. However, the mechanisms of uterine regeneration are poorly understood. Elucidation of uterine regeneration will benefit efforts to establish a novel therapeutic approach for serious obstetric complications, such as infertility, recurrent pregnancy loss, and uterine rupture. An overly thin endometrium is one of the reasons for implantation failure and recurrent pregnancy loss, which sometimes results from intrauterine adhesions after surgical procedures, such as dilation and curettage of the uterine cavity (1). Uterine rupture, a life-threating condition for both mother and baby, is often caused by the disruption of uterine surgical scarring derived from cesarean section or myomectomy with uterine distension caused by fetal growth. This disease may be associated with poor wound healing of the myometrium after uterine surgery (2). To date, these serious issues in pregnancy have no effective resolutions, although medical research for uterine reconstruction and regeneration may offer solutions. Thus, establishment of a novel approach to understand uterine regeneration is an urgent task in today's obstetrical basic research.

We recently reported a novel technique of uterine decellularization in a rat model (3). Notably, the rat uterus was partially reconstructed after the transplantation of uterine scaffold decellularized by the 
A

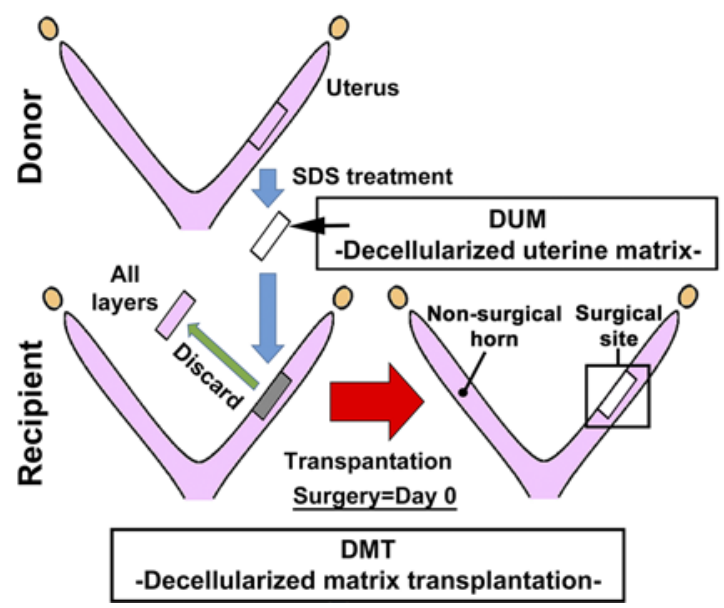

B

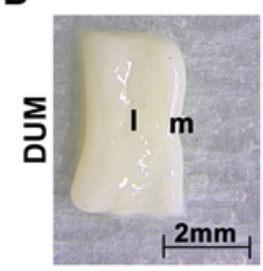

C

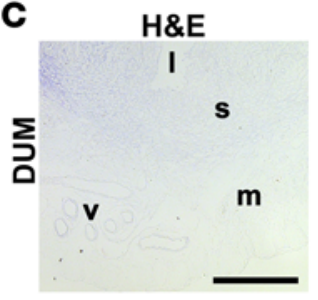

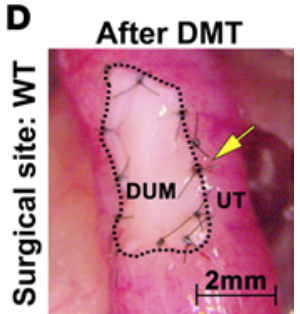

Day 0

Figure 1. Experimental procedures of a mouse DMT model. (A) A schematic illustration of a mouse model of decellularized matrix transplantation (DMT). (B) A macroscopic image of a decellularized uterine matrix (DUM) from a donor mouse. I, luminal surface; m, myometrial layer. Scale bar: 2 mm. (C) H\&E staining of a DUM before transplantation. Intact cells were totally absent, and the original uterine structure was sustained in DUM. I, lumen; s, stromal layer; m, myometrial layer; v, vascular structure. Scale bar: $200 \mu \mathrm{m}$. (D) A macroscopic image of the transplantation site in a recipient mouse uterus immediately after DMT (day 0). A trimmed DUM was exactly fitted into a rectangular defect in a recipient mouse uterus and fixed to the recipient uterus with intermittent sutures. UT, a recipient uterus surrounding DUM; yellow arrow, a 10-0 nylon suture thread; dotted line, a boundary between DUM and UT. Scale bar: $2 \mathrm{~mm}$.

treatment of SDS or high hydrostatic pressure. By developing this technology in the current study, we established a mouse model of uterine reconstruction and regeneration by decellularized matrix transplantation (DMT), in which decellularized uterine tissues from recipient mice are transplanted into artificially induced defects of recipient mouse uteri.

STAT3 is a transcription factor crucially involved both in epithelial proliferation during regeneration of many different tissues and in maintenance of pregnancy, especially during embryo implantation (4-6). In the current study, we elucidated the role of STAT3 in uterine epithelial regeneration in the DMT mouse model utilizing Stat3 conditional knockout mice. Here, we report that uterine epithelial reconstruction controlled by STAT3 contributes to uterine regeneration.

\section{Results}

Uterine reconstruction in a mouse DMT model. To develop a new strategy of uterine reconstruction, we established a mouse model using the DMT procedure, in which SDS-treated decellularized uterine matrices (DUMs) were transplanted into the artificially induced rectangular defects in recipient mouse uteri (Figure 1A). As macroscopic and microscopic findings, SDS-treated DUMs did not have any intact cells or nuclei but maintained the matrix structure of a normal uterus, including the luminal surface, stroma, myometrium, and blood vessels (Figure 1, B and C). These findings indicate the suitability of the DUM as an extracellular matrix (ECM) scaffold for uterine regeneration. As shown in Figure 1D, immediately after DMT (day 0), the trimmed DUM was exactly fitted into the rectangular defective region in a recipient mouse uterus and fixed to the recipient uterus with intermittent sutures. On day 28 , the transplanted DUM macroscopically looked similar to the original recipient uterus around the DUM, and contained newly formed vessels (Figure 2A). Histological analysis revealed that all the uterine layers, including the luminal epithelium, the glandular epithelium, the stroma, and the myometrium, were reconstructed in the transplanted DUM on day 28 (Figure 2B).

In the immunohistochemical analysis, normal immunoreactivity for an epithelial marker (cytokeratin 8 [CK8]), a myometrial marker ( $\alpha$-smooth muscle actin [ $\alpha \mathrm{SMA}]$ ), and ovarian hormone receptors (estrogen receptor $\alpha[E R \alpha]$ and progesterone receptor [PR]) was observed in the reconstructed uterus (Figure 2C). Since Rosa26 Cre-reporter knockin (R26GRR) mice exhibit green emission before and red emission after Cre-mediated recombination (7), and since Amhr2-Cre mice have specific expression of Cre recombinase in the stroma and myometrium (8), we generated R26GRR/Amhr2-Cre mice, which have stroma/myome- 
A

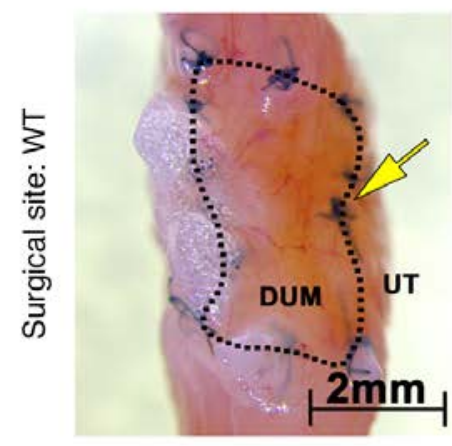

Day 28
B

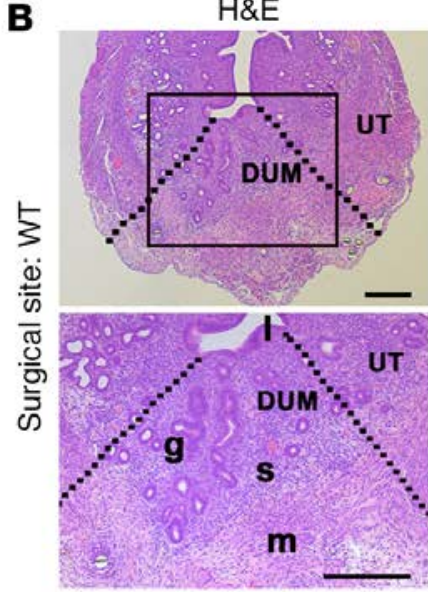

Day 28

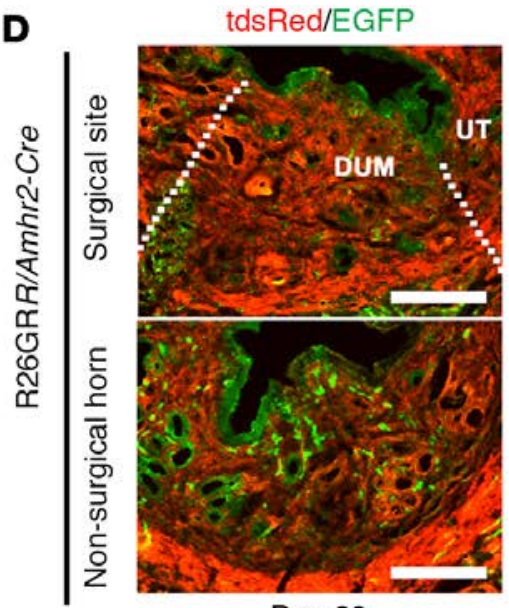

Day 28

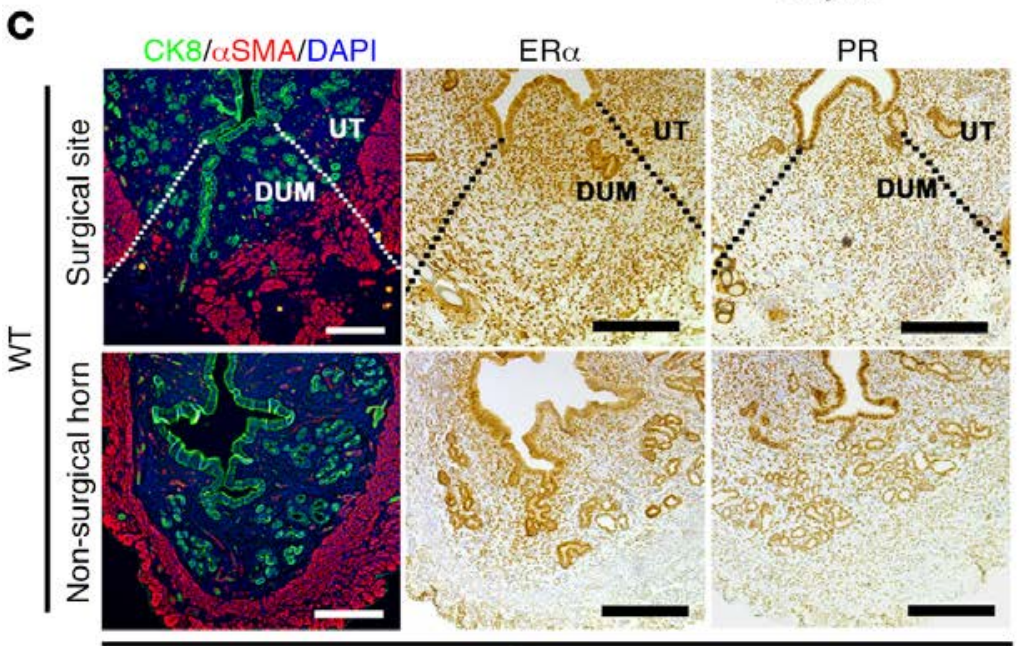

Day 28

Figure 2. Mouse uterine reconstruction by DMT. (A) A macroscopic image of the transplantation site of decellularized uterine matrix (DUM) on day 28. The reconstructed uterine tissue with newly formed vessels was observed within the DUM. UT, a recipient uterus surrounding DUM; yellow arrow, a 10-0 nylon suture thread; dotted line, a boundary between DUM and UT. Scale bar: $2 \mathrm{~mm}$. (B) H\&E staining of the transplantation site of DUM on day 28. All the uterine layers, including the luminal epithelium, the glandular epithelium, the stroma, and the myometrium, were regenerated in DUM. I, luminal epithelium; g, glandular epithelium; s, stroma; m, myometrium. Scale bar: $200 \mu \mathrm{m}$. (C) Immunostaining of cytokeratin 8 (CK8), $\alpha$-smooth muscle actin ( $\alpha$ SMA), estrogen receptor $\alpha(E R \alpha)$, and progesterone receptor (PR) in the transplanted DUM and the nonsurgical horn on day 28 . Immunoreactivity for these proteins was similarly observed both in the transplanted DUM and the nonsurgical horn. Scale bar: $200 \mu \mathrm{m}$. (D) Fluorescence assay of R26CRR/Amhr2-Cre recipient uteri on day 28. The newly formed epithelium had green fluorescence, and the stroma and myometrium had red fluorescence in the transplanted DUM, indicating that the newly formed epithelium in the DUM is the progeny of the original recipient epithelium and the regenerated stroma and myometrium possess normal uterine mesenchymal property. Each image is a representative from at least 3 independent experiments. Scale bar: $200 \mu \mathrm{m}$.

trium-specific red fluorescence of tdsRed in the uterus, to identify cell lineage of DMT. We transplanted the DUMs from WT donor mice into R26GRR/Amhr2-Cre recipient mice. In the R26GRR/Amhr2-Cre uterus, mesenchymal cells, including stromal and myometrial cells with Amhr2 expression, produce Cre recombinase that induces tdsRed red fluorescence. Epithelial cells without Amhr2 expression manifested EGFP fluorescence, which was demonstrated in the nonsurgical horns of R26GRR/Amhr2-Cre recipient mice (Figure 2D). Importantly, we found green fluorescence in the newly formed epithelium and red fluorescence in the stroma and myometrium in the transplanted DUMs on day 28 (Figure 2D). These findings suggest that the regenerated stroma and myometrium in the DUM possess normal uterine mesenchymal properties and the newly formed epithelium in the DUM originates from the recipient epithelium.

We verified functions of the reconstructed uterus by evaluating not only uterine receptivity around the time of implantation, but also pregnancy outcome at full term. We previously reported that proliferation-differentiation switching, cessation of epithelial proliferation, and acceleration of stromal proliferation in the uterus on day 4 of pregnancy (vaginal plug = day 1 of pregnancy) is a marker of uterine 
A

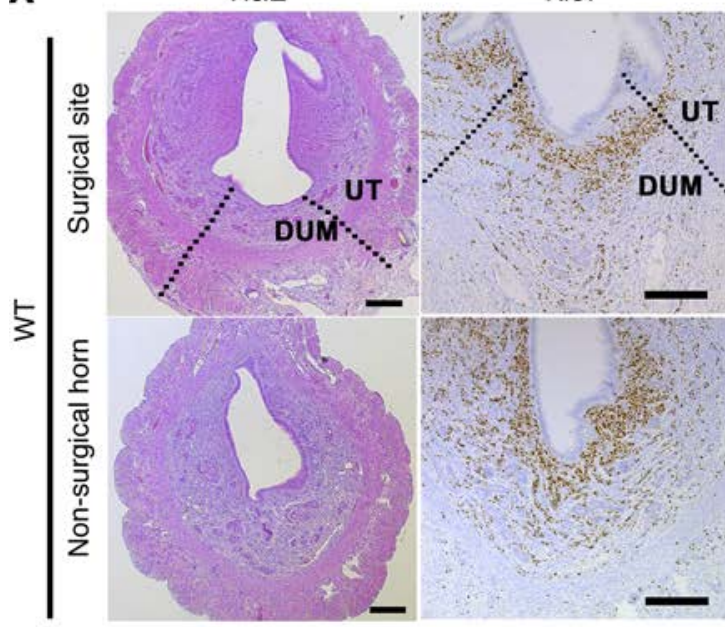

Day 4 of pregnancy

D

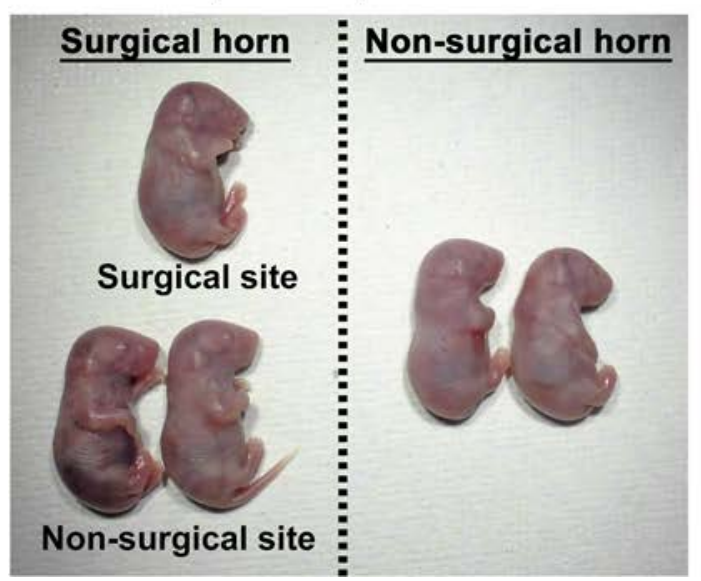

B

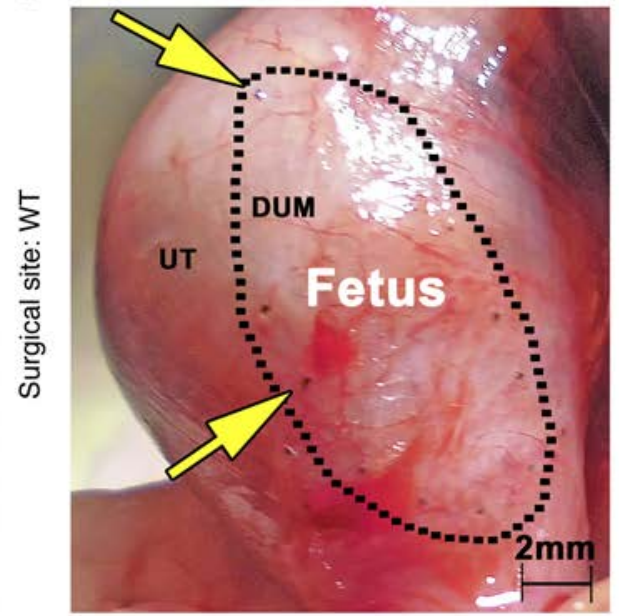

Day 19 of pregnancy
C

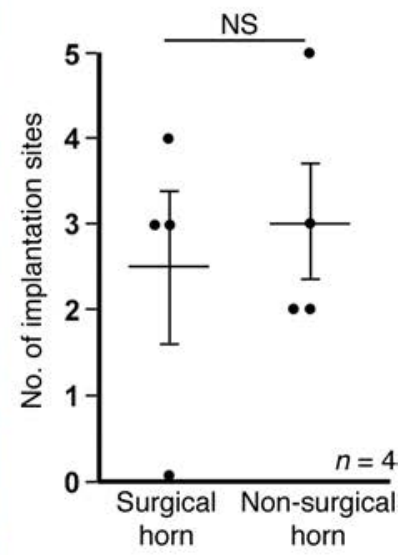

E



Figure 3. Regenerated uteri contribute to successful pregnancy. (A) H\&E staining and Ki67 immunostaining of the recipient mouse uteri on day 4 of pregnancy. Normal proliferation-differentiation switching during implantation, a marker of uterine receptivity, was observed in the transplanted decellularized uterine matrix (DUM), indicating the normal capacity for embryo implantation in the regenerated uterus. WT recipient mice 1 month after decellularized matrix transplantation were crossed with fertile WT males. Scale bar: $200 \mu \mathrm{m}$. UT, a recipient uterus surrounding DUM; dotted line, a boundary between DUM and UT. Each image is a representative from at least 3 independent experiments. (B) A macroscopic image of an implantation site at the transplanted DUM site on day 19 of pregnancy. To assess pregnancy outcome of WT recipient mice, cesarean section was performed on day 19 of pregnancy. The reconstructed uterus within the transplanted DUM site showed normal appearance and looked like a pregnant uterus on day 19. Yellow arrow, a $10-0$ nylon suture thread. Scale bar: $2 \mathrm{~mm}$. (C) Numbers of implantation sites in the surgical and nonsurgical horns in the pregnant recipient mice on day 19 of pregnancy. Numbers of implantation sites in the pregnant recipient mice were comparable between the surgical and nonsurgical horns. (D) A macroscopic image of pups in the surgical and nonsurgical horns of the same recipient mouse delivered by cesarean section on day 19 of pregnancy. Size and general appearance of pups at the transplanted DUM sites were similar to those of pups at the nonsurgical site as well as those of pups in the surgical horns. (E) Weight of pups that developed at the surgical site, the nonsurgical site in the surgical horn, and the nonsurgical horn on day 19 of pregnancy. The weight of pups that developed at the transplanted DUM sites were similar to those of pups that developed at the nonsurgical site as well as those of pups that developed in the surgical horns, indicating that regenerated uterine capacity was normal and could maintain full pregnancy.

receptivity in embryo implantation (9). We crossed WT recipient mice 1 month after DMT with fertile WT males and collected the uteri on day 4 of pregnancy. Immunostaining of Ki67, a cell proliferation marker, revealed that the regenerated uterus at the site of transplanted DUM had normal proliferation-differentiation switching, which was comparable to the nonsurgical horn (Figure 3A). To assess pregnancy outcome in WT recipient mice, we performed cesarean sections on day 19 of pregnancy. Among 5 recipient mice with vaginal plugs, 4 mice became pregnant. Three of four pregnant mice had implantation sites at the sites of transplanted DUMs (Figure 3B), and one of them had two implantation sites at the DUM site. Macroscopic analysis of implantation sites at the DUM sites showed normal appearance; uteri with DUM sites looked like pregnant day 19 uteri without increase of resorption (Figure 3B). Numbers of implanta- 
A

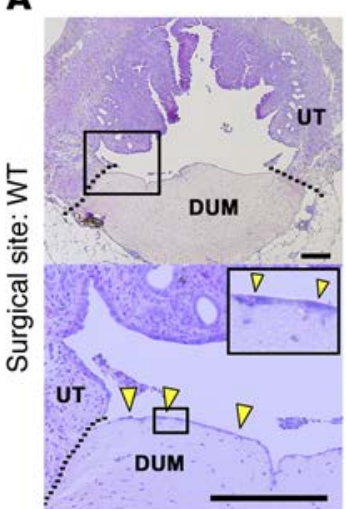

Day 1

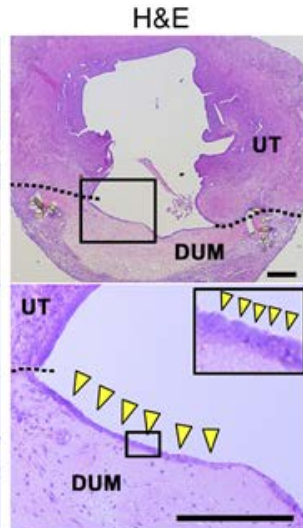

Day 3

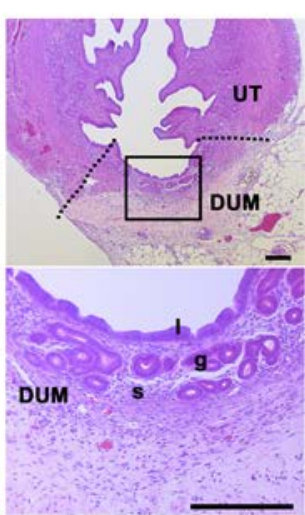

Day 7
B

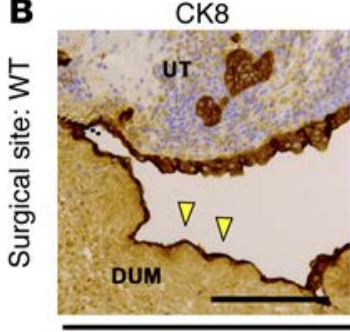

C tdsRed/EGFP

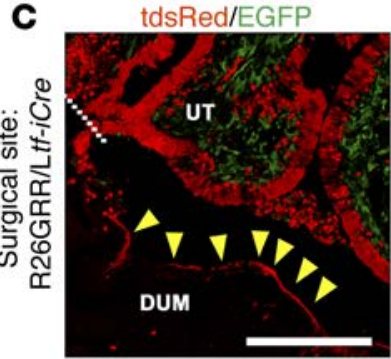

Day 3
$\mathrm{ER} \alpha$

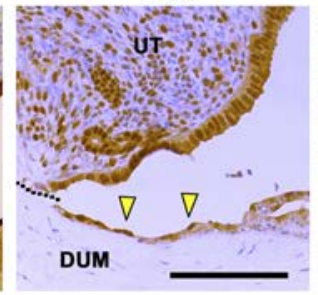

Day 1
PR

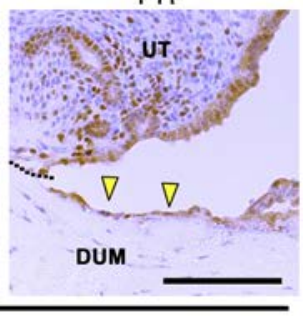

DUM
Figure 4. Early phase of uterine reconstruction process in DMT.

(A) An early phase of uterine reconstruction process in the transplanted decellularized uterine matrices (DUMs) was assessed by $H \& E$ staining. Flat cells rapidly migrated onto the DUM on day 1 , and they increased in number and became taller on day 3 . Luminal epithelium was fully reconstructed, and stromal regeneration with glandular structure was also observed by day 7. Yellow arrowheads, cells constituting regenerating epithelium on DUM. (B) Immunostaining of cytokeratin 8 (CK8), estrogen receptor $\alpha$ $(E R \alpha)$, and progesterone receptor (PR) in the transplanted DUM on day 1 . Flat cells on DUM exhibited prominent immunoreactivity for these proteins. Yellow arrowheads, flat cells constituting the regenerating epithelium on DUM. (C) Fluorescence assay of the transplantation site in the R26GRR/Ltf-iCre recipient uterus on day 3. Regenerating epithelium expressed tdsRed, indicating that it was the progeny of neighboring epithelial cells in UT. UT, a recipient uterus surrounding DUM; I, luminal epithelium; g, glandular epithelium; s, stroma; yellow arrowheads, cells constituting regenerating epithelium on DUM; dotted line, a boundary between DUM and UT. Each image is a representative from at least 3 independent experiments. Scale bar: $200 \mu \mathrm{m}$.

tion sites in the recipient mice were comparable between the surgical and nonsurgical horns (Figure 3C). The size, general appearance, and weight of pups that developed at the transplanted DUM sites were similar to those of pups that developed at the nonsurgical site or the surgical horns (Figure 3, D and E). Taken together, our findings strongly support DMT as a method to enable uterine regeneration and recover uterine function.

Uterine luminal epithelium possesses remarkable regeneration capacity. To clarify the mechanism of uterine regeneration, we next histologically examined the processes of uterine reconstruction at the surgical site. $\mathrm{H} \& \mathrm{E}$ staining showed that flat cells rapidly migrated onto the surface of DUM and sealed the entire surface as early as day 1 (Figure 4A). On day 3 , the flat cells became taller and increased in number (Figure 4A). A normal-appearing luminal epithelium was fully reconstructed by day 7 (Figure 4A). Distinct immunoreactivity of $\mathrm{CK} 8, \mathrm{ER} \alpha$, and PR in the regenerating luminal epithelium on day 1 demonstrated that regenerating flat cells possess uterine epithelial competency, even in the earlier regenerative phase (Figure 4B). To confirm the epithelial lineage origin of the flat cells in the early regenerating phase, we combined another cell lineage tracing assay with the present DMT model, in which WT DUM was transplanted into R26GRR/Ltf-iCre recipient mice. $L t f$ is an estrogen-inducible gene specific to the uterine epithelium. In the R26GRR/Ltf-iCre uterus, epithelial cells expressing Ltf produce Cre recombinase, which induces tdsRed red fluorescence, and stromal cells lacking Ltf expression showed EGFP green fluorescence. Fluorescence assay showed red fluorescence in the regenerating diestrus luminal epithelium on day 3, proving that these migrating flat cells on DUMs are progenies of the recipient epithelial cells forming the nascent luminal epithelium later (Figure 4C). These results were comparable to the observations in R26GRR/Amhr2-Cre mice. As for regeneration of the glandular epithelium, H\&E staining of serial sections on day 3 revealed that initial superficial flat cells formed a gland-like structure in the transplanted DUMs, indicating migration and invagination of luminal epithelial cells (Supplemental Figure 1A; supplemental material available online with this article; doi:10.1172/jci.insight.87591DS1). On day 7, the normal-appearing mature glandular epithelium with taller cells was increased (Supplemental Figure 1B and Supplemental Figure 2). In contrast, stromal regeneration was not observed by day 3, but it was present on day 7 (Figure 4A and Supplemental Figure 2), suggesting that stromal regeneration capacity is relatively lower than that of the luminal and glandular epithelium. These findings indicate that regeneration of the luminal epithelium is followed by the glandular epithelium and then the stroma. 
A

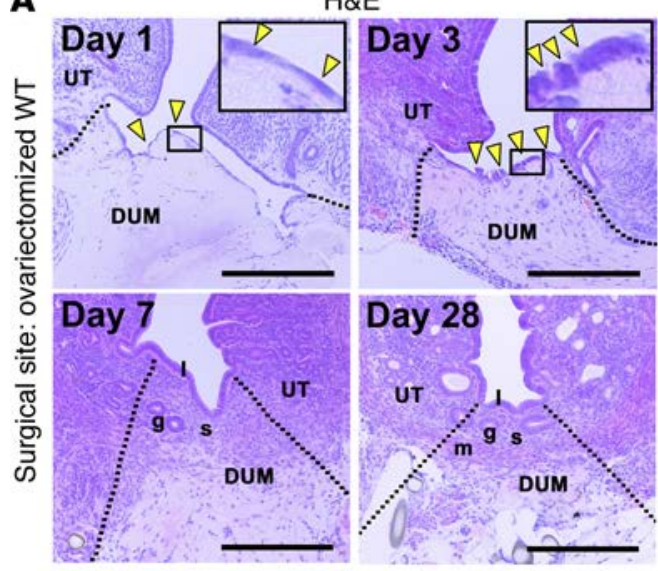

B

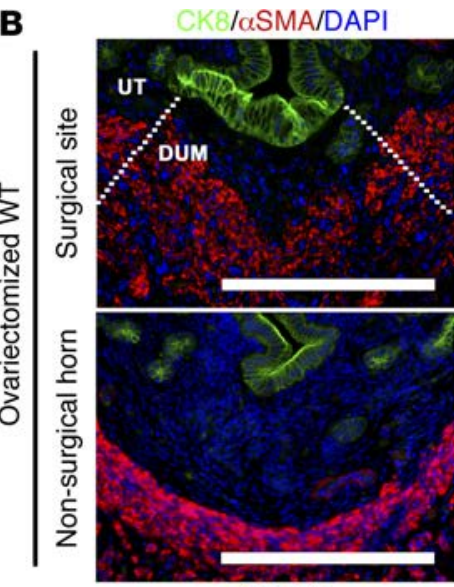

Day 28

Figure 5. Uterine reconstruction process is unchanged in the absence of ovarian hormones. (A) An early phase of uterine reconstruction process in the transplanted decellularized uterine matrices (DUMs) of ovariectomized recipient uteri was assessed by $\mathrm{H} \& \mathrm{E}$ staining. The process of uterine reconstruction and regenerated tissue structure in the ovariectomized mouse DMT model was comparable to that in a mouse DMT model without ovariectomy. Yellow arrowheads, cells constituting regenerating epithelium on DUM. (B) Immunostaining of cytokeratin 8 (CK8) and $\alpha$-smooth muscle actin ( $\alpha$ SMA) in the ovariectomized mouse DMT model. Normal immunoreactivity for CK 8 and $\alpha$ SMA were observed in the transplanted DUM on day 28. UT, a recipient uterus surrounding DUM; I, luminal epithelium; g, glandular epithelium; s, stroma; m, myometrium; yellow arrowheads, cells constituting regenerating epithelium on DUM; dotted line, a boundary between DUM and UT. Each image is a representative from at least 3 independent experiments. Scale bar: $200 \mu \mathrm{m}$.

Ovarian hormones are not essential for uterine reconstruction. Two ovarian hormones, estrogen and progesterone, regulate cyclic changes in the uterus to prepare for embryo implantation (10-13). In humans, estrogen secreted from ovarian follicles enhances the growth of the endometrium and progesterone secreted from the corpus lutea leads to predecidual changes, while the withdrawal of ovarian hormones induces shedding of the endometrium, namely, menstruation. The influence of ovarian hormones on the mouse uterus is similar to that in humans in many aspects (12). To assess effects of ovarian hormones on uterine reconstruction, we next employed the ovariectomized mouse DMT model. In this model, the recipient mice were ovariectomized 2 weeks before DMT induction, and the DUMs from ovariectomized donor mice were transplanted. Histological analysis revealed that the process of uterine reconstruction was comparable between the mouse models with and without ovariectomy (Figure 4A and Figure 5A), and the regenerated tissue structure in the transplanted DUM was also similar between the two models on day 28 (Figure 2B and Figure 5A). Immunoreactivity for CK8 and $\alpha$ SMA in the transplanted DUM looked similar to that in the nonsurgical horn on day 28 in the ovariectomy model, which was also a comparable finding to the nonovariectomy model (Figure 5B). These findings indicate that ovarian hormones are dispensable for uterine reconstruction in the mouse model of DMT.

Uterine epithelial regeneration is promptly initiated without estrogen. We next focused on uterine epithelial regeneration, especially its induction mechanism, which primarily triggers uterine reconstruction. We examined the very early stage of uterine regeneration in the ovariectomized mouse DMT model by 24 hours after DMT (DMT-24h = day 1). Ki67 immunostaining revealed that epithelial proliferation surrounding the DUM increased by DMT-12h and was more prominent at DMT-24h (Figure 6A), suggesting that proliferating epithelial cells may cover the surface of the DUM rapidly. These results also indicate that uterine epithelial regeneration does not require estrogen, which is the well-known inducer of uterine epithelial proliferation in vivo $(14,15)$. To evaluate the impact of estrogen on the uterine regeneration mechanism, we employed subcutaneous injections with $100 \mathrm{ng}$ of $17 \beta$-estradiol $\left(\mathrm{E}_{2}\right)$ or the vehicle 6 hours before DMT in the ovariectomy model. In the vehicle group, few cells were observed on the DUM at DMT-6h. The flat epithelial cells started to migrate onto the DUM at DMT-12h and afterwards increased in number. The DUM was completely covered by new epithelium at DMT-72h (day 3) (Figure 6B). $E_{2}$ treatment neither promoted the epithelial regeneration process nor increased even the cellularity of a newly formed epithelium by DMT-72h (Figure 6B). These findings indicate that a yet 
A

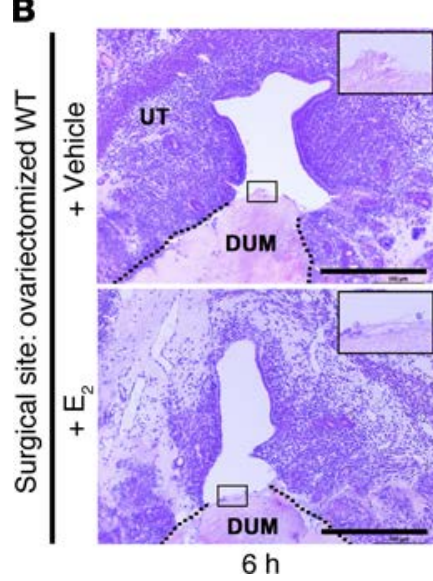

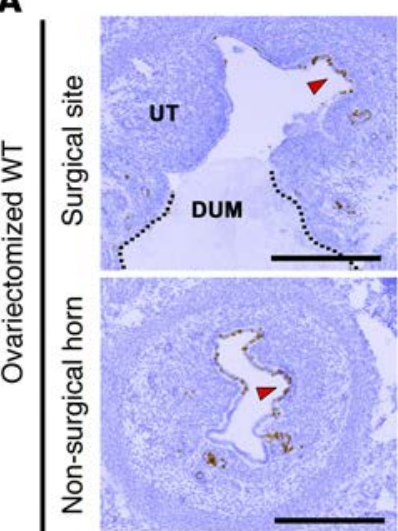

$6 \mathrm{~h}$

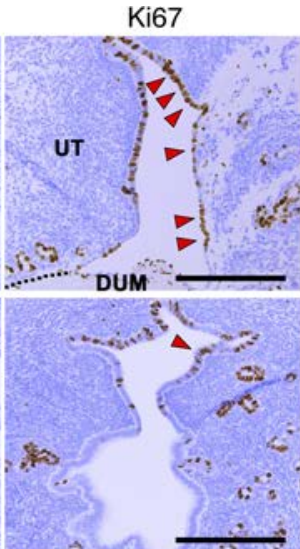

$12 \mathrm{~h}$

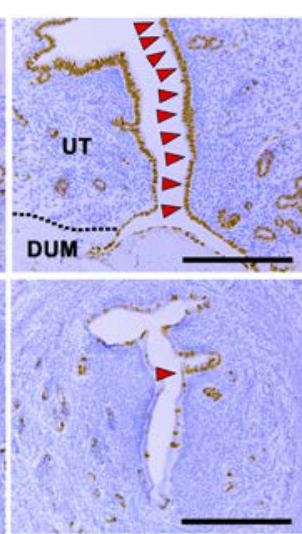

$24 \mathrm{~h}$

(= day 1 )

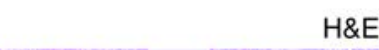

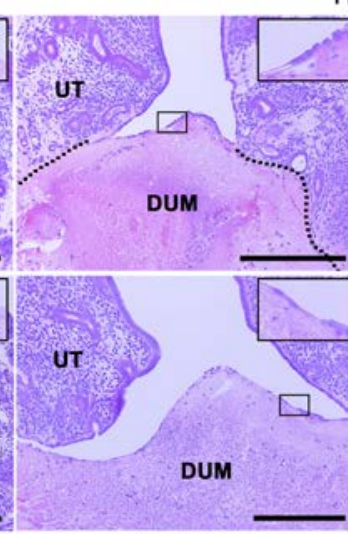

$12 \mathrm{~h}$
$\mathrm{H} \& \mathrm{E}$

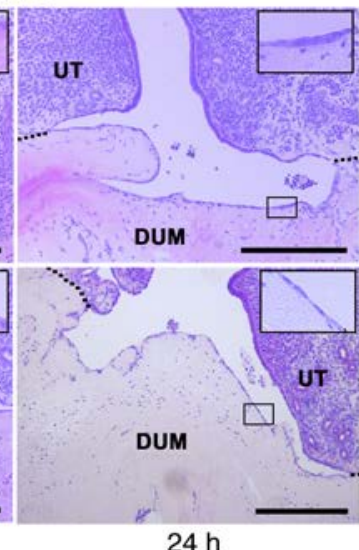

Figure 6. Uterine epithelium rapidly initiates regeneration independently of estrogen. (A) Ki67 staining of the recipient uteri 6 , 12 , and 24 hours after DMT in the ovariectomized mouse DMT model without 17 $\beta$-estradiol $\left(E_{2}\right)$ treatment. Upregulation of cell proliferation in the epithelium of the recipient uterus surrounding decellularized uterine matrix (DUM) started 12 hours after DMT (DMT-12h), which became more prominent at DMT-24h. Red arrowheads, proliferating epithelial cells in a recipient uterus surrounding DUM. (B) An early phase of uterine reconstruction process in the transplanted DUMs of ovariectomized recipient uteri with or without $\mathrm{E}_{2}$ was assessed by H\&E staining. In both groups, few flat cells were observed at DMT-6h, and they increased in number afterward, finally reconstituting new epithelium at DMT-72h. No apparent difference was observed between the two groups. Dotted line, a boundary between DUM and UT. UT, a recipient uterus surrounding DUM. Each image is a representative from at least 3 independent experiments. Scale bar: $200 \mu \mathrm{m}$.

unidentified biological mechanism controls uterine epithelial regeneration, even without estrogen, and its impact overwhelms estrogen influence.

STAT3 is a key player for uterine epithelial regeneration. To further investigate the uterine epithelial regeneration, we targeted a specific molecule involved in cell proliferation. It was reported that STAT3 is responsible for epithelial proliferation during tissue regeneration and maintenance in other organs (16-26). As for its function in the uterus, there has been limited information, except the evidence that uterine conditional deletion of Stat 3 leads to implantation failure $(4,5)$. Therefore, we examined the role of STAT3 in uterine epithelial regeneration, using an ovariectomized DMT mouse model. Nuclear accumulation of phosphorylated STAT3 (pSTAT3) was specifically observed only nearby the surgical site, especially in the epithelial cells surrounding DUM. It was initiated as early as at DMT-6h and became more prominent at DMT-24h (Figure 7A). To assess the function of STAT3 in epithelial regeneration, we next used mice with uterine deletion of Stat3 $(\triangle S t a t 3)$. We first confirmed the efficient inhibition of pSTAT3 accumulation into nuclei at the surgical sites in $\triangle$ Stat 3 mice (Figure $7 \mathrm{~B}$ ). Subsequently, we found that the regenerating epithelium on DUMs at DMT-24h had fewer cells in $\triangle$ Stat 3 mice than that in WT mice (Figure 7, B and C). We also found significantly retarded proliferative activity in the epithelial cells surrounding the DUM in $\triangle$ Stat 3 mice compared with WT mice (Figure 7, B and D), as evident from the reduced number of cells in the regener- 
A

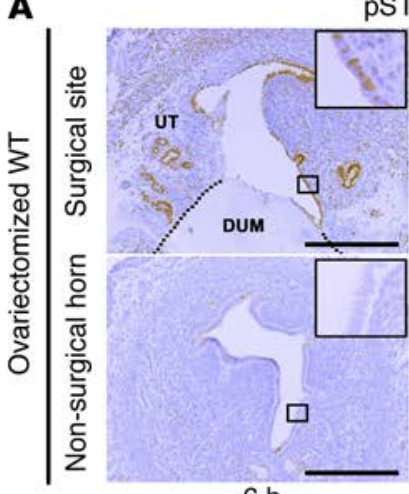

$6 \mathrm{~h}$
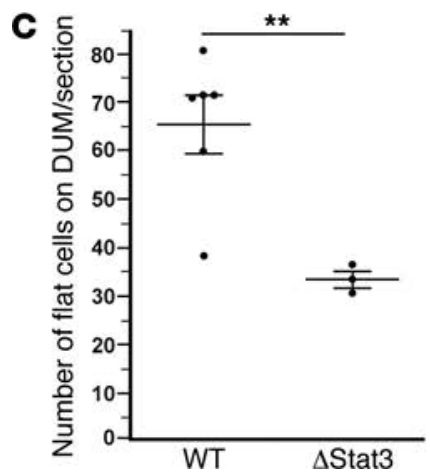

pSTAT3

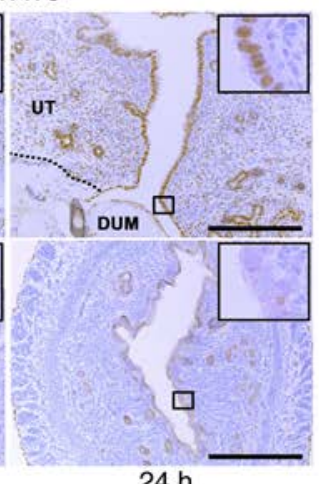

$24 \mathrm{~h}$
B

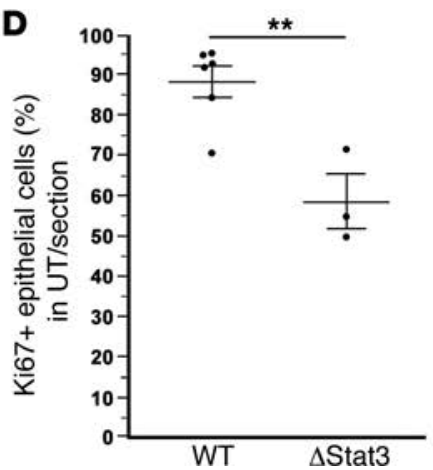

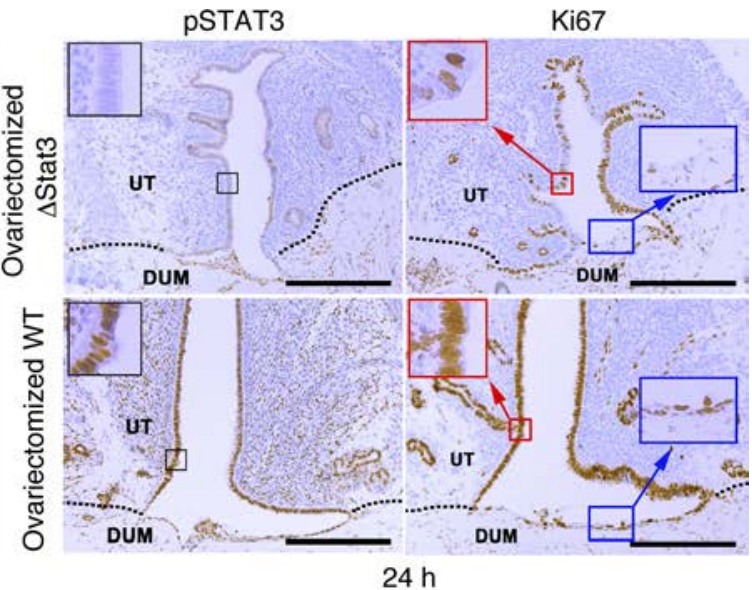

Figure 7. STAT3 modulates uterine epithelial regeneration. (A) Immunostaining of phosphorylated STAT3 (pSTAT3) in the ovariectomized mouse DMT model. Immunoreactivity for pSTAT3 was observed primarily in the epithelium of UT at DMT-6h and became more intense at DMT-24h. (B-D) Evaluation of epithelial regeneration comparing $\triangle$ Stat3 and WT mice. (B) Negative staining of pSTAT3 in the $\triangle$ Stat3 mouse uterus ensured efficient knockout of uterine Stat3. (B and C) The number of flat cells on the decellularized uterine matrix (DUM) that reconstitute the epithelium was significantly decreased in $\Delta$ Stat3 mice ( ${ }^{*} P<0.01$ ), and (B and $\mathbf{D}$ ) the percentage of Ki67-positive epithelial cells in the recipient uterus surrounding DUM was significantly lower in $\triangle S$ Stat3 mice than that in WT mice ( ${ }^{*} P<0.01$ ), suggesting the significant retardation of epithelial regeneration due to uterine deletion of Stat3 ( $\left.\triangle S t a t 3\right)$. UT, a recipient uterus surrounding DUM; dotted line, a boundary between DUM and UT. Each image is a representative from at least 3 independent experiments. Scale bar: $200 \mu$ m.

ating epithelium. These findings indicate that STAT3 mediates cell proliferation upon injury and increases the cell pool used to cover the disrupted surface. Taken together, our results provide evidence that STAT3 is critical for uterine epithelial regeneration.

STAT3 is activated during uterine reconstruction process in a physiological setting. To evaluate whether STAT3 contributes to uterine epithelial regeneration in physiological context, we examined pSTAT3 expression not only in the postpartum mouse uterus, but also in the human uterus in the menstrual and early proliferative phases when the cyclic process of endometrial reconstruction occurs (27-30). We found abundant nuclear accumulation of pSTAT3 in the mouse uterine glandular and luminal epithelium after delivery (Figure 8A), although few epithelial cells had immunoreactivity of pSTAT3 in the nonpregnant mouse uterus in the diestrus phase. The human uterus also exhibited abundant nuclear accumulation of pSTAT3, although few epithelial cells showed pSTAT3 immunoreactivity during the late secretory phase (Figure $8 \mathrm{~B})$. These observations suggest that acute transient inflammation occurring during parturition and menstruation induces uterine STAT3 phosphorylation, which promotes uterine reconstruction. In addition, pSTAT3 had low intensity in the stroma and the myometrium in the uterine reconstruction stage of both mice and humans (Figure 8), indicating similar mechanisms of uterine reconstruction in both species.

\section{Discussion}

In the present study, we have established an experimental procedure, the mouse model of DMT, in which transplantation of SDS-treated DUMs into the artificially induced defects of recipient uteri enables reconstruction of functional uteri. This mouse model has revealed that uterine epithelial regeneration rapidly occurs after DMT, which is impaired by STAT3 deficiency. These findings indicate that the DMT mouse model is a useful tool to understand uterine regeneration. 
A

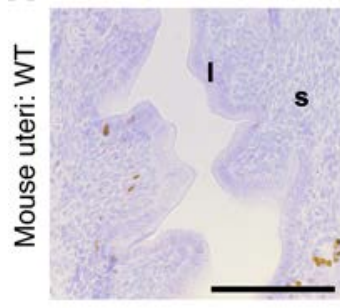

Diestrus
pSTAT3



B

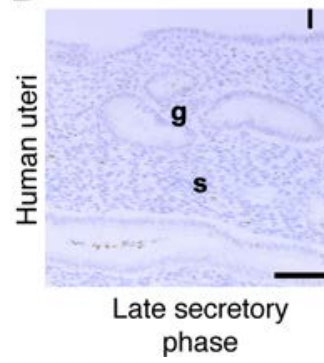

pSTAT3

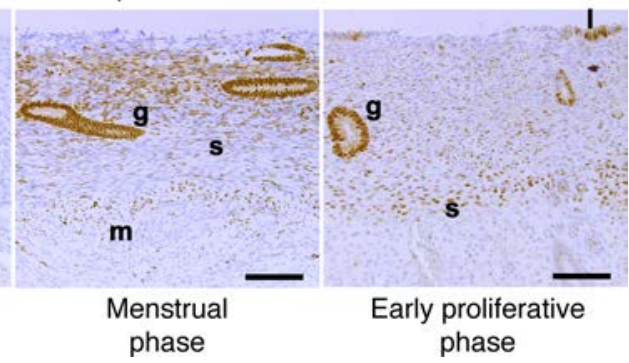

Figure 8. Expression of pSTAT3 in the physiological uterine regeneration process. (A) Immunostaining of phosphorylated STAT3 (pSTAT3) in the nonpregnant and postpartum mouse uteri. As for the nonpregnant sample, a diestrus uterus was used. Immunoreactivity for PSTAT3 was observed in the luminal and glandular epithelium after delivery, in contrast to its negative staining in the nonpregnant uterus. (B) Immunostaining of pSTAT3 in the human uterus at the late secretory, menstrual, and early proliferative phases. Immunoreactivity for pSTAT3 was observed in the luminal and glandular epithelium at the menstrual and early proliferative phases, as opposed to the negative staining during the late secretory phase. I, luminal epithelium; g, glandular epithelium; s, stroma; $m$, myometrium. Each image is a representative from at least 3 independent experiments. Scale bar: $100 \mu \mathrm{m}$.

Decellularization techniques have been used for tissue reengineering research in various organs, such as heart, lung, liver, ovary, and so on (31-35). The advantage of our decellularized technique is the preservation of the physiological ECM structure, composition, and stiffness specific to each organ, which is considered an essential factor for tissue-specific cell differentiation (36-42). Many studies using this decellularization technique also utilized the recellularization technique, in which cells are seeded into decellularized tissues by injection or perfusion of cell suspension and cultured for several weeks in vitro; through this combination method, organs partially regain their original functions (31-35). By contrast, our model transplants decellularized uteri into living mice, thereby enabling analysis of the in vivo regeneration process.

Since regeneration prominently occurs at the site of the transplanted DUM, we believe that this model can reveal the upregulated molecular signaling associated with uterine regeneration. By comparison with the nonsurgical uterine horn in which regeneration occurs poorly, essential molecular pathways responsible for uterine reconstruction can be extracted. In addition, the DMT model visualizes two distinct parts in the surgical site: the cell-supplying compartment surrounding the DUM and the regenerating compartment in the DUM. Moreover, we can apply the genetically modified mice to the DMT model. These aspects of the DMT model enable us to evaluate the uterine regeneration process precisely, as shown in the present study in which genetically modified mice were used to reveal a significant role of STAT3. To date, the role of STAT3 has only been elucidated in the context of pregnancy, especially in embryo implantation. It has been reported that STAT3 mediates signaling from leukemia inhibitory factor (LIF) during implantation and plays a crucial role for implantation, because both Lif-deficient mice and $\Delta$ Stat3 mice show implantation failure $(4,5,43)$. Implantation failure occurs in $\triangle$ Stat 3 mice because of aberrantly enhanced estrogenic influence on the preimplantation uterus (4), but the specific mechanism that causes poor uterine conditions remains unknown. Here we have discovered the role of STAT3 in the context of uterine epithelial regeneration. It is possible that disruption of uterine regeneration due to lack of Stat3 may be associated with implantation failure.

Rapid promotion of epithelial cell proliferation upon tissue damage is observed in many organs. Recent evidence suggested that acute inflammation can be a candidate for induction of epithelial proliferation and regeneration. For example, intestinal epithelial cells rapidly start proliferation upon injury and, subsequently, Lgr5 ${ }^{+}$intestinal stem/progenitor cells are activated (44). Ovarian surface epithelial (OSE) cells initiate proliferation upon the disruption of the ovarian surface after ovulation, which is considered an inflammatory-like physiological process, when the number of proliferating OSE stem/progenitor cells increases (45). The JAK/STAT pathway is a major pathway that mediates cytokine signaling and controls cell survival, death, differentiation, and proliferation (46). In the JAK/STAT pathway, STAT3 is one of the 
major effectors that connects inflammation to cell proliferation $(16,19,46-49)$. STAT3 transduces signals as a transcriptional factor by its phosphorylation and nuclear entry upon stimulation of IL-6 family cytokines, such as IL-6, IL-11, LIF, and oncostatin-M, secreted by activated leukocytes during inflammation. The increase in nuclear accumulation of pSTAT3 in the DMT model may reflect acute inflammation at the surgical site, and the retardation of epithelial regeneration due to uterine depletion of Stat3 strongly suggests the important function of STAT3 in connecting acute inflammation to uterine epithelial regeneration.

In human and nonhuman primates, the upper and lower sides of the endometrium are called the functional and basal layers, respectively $(27,50)$. The functional layer containing the glandular/luminal epithelium and the stroma is shed and regenerates every menstrual cycle $(27,50)$. The rodent endometrium also undergoes regular dynamic remodeling, which intensively occurs during parturition, accompanied by endometrial bleeding, although the function of the basal layer of endometrium is unclear in rodents (51). Thus, the endometrium, regardless of species and presence of menstruation, has a high regenerative capacity. Our observation that similar localization of endometrial pSTAT3 occurred in both mice after parturition and humans after menstruation suggests that there are common mechanisms of uterine regeneration in mice and humans. Since neutrophils have key roles in endometrial repair of mice and humans (52-54), it is possible that neutrophil-derived cytokines may activate STAT3 and further induce epithelial regeneration in the early regeneration stage.

About $50 \%$ of proliferating epithelial cells around the DUMs in $\Delta$ Stat 3 mice may indicate not only the importance of STAT3 on uterine epithelial regeneration, but also the presence of other pathways responsible for uterine epithelial regeneration. Indeed, a recent study identified gp130/Src/YAP signaling as a responsible pathway that links inflammation upon damage to intestinal epithelial regeneration independently of STAT3 (16). Further molecular analyses using the DMT model will provide new insights into the molecular mechanism of uterine epithelial regeneration.

What is the driving force of epithelial cell regeneration in a disrupted surface in the uterus? We hypothesized that epithelial cells rapidly lose polarity and initiate migration onto the disrupted surface and proliferation to supply cells upon injury, which is called "epithelial restitution" $(16,55)$. Since "epithelial restitution" is a highly conserved mechanism in various organs such as small intestine and colon against tissue damage, it may be involved in the machinery of uterine luminal epithelial regeneration in our mouse model. These hypotheses may also account for the pathogenesis of adenomyosis, in which endometrium-like tissues are ectopically generated in the myometrial layer. Epidemiological evidence has revealed the relationship between this disease and uterine traumatic procedures, such as cesarean section and intrauterine curettage. More precisely, uterine epithelial cells may rapidly invade the damaged site in the myometrial layer due to surgical injury and then ectopically form ectopic endometrium-like lesions. Further investigations are needed to elucidate these hypotheses in terms of the pathogenesis of adenomyosis.

The mechanisms of glandular regeneration may be complicated, and it is suggested that one of the possible mechanisms is "epithelial invagination." "Epithelial invagination" is a reported phenomenon in the intestinal healing process subsequent to "epithelial restitution," in which epithelial cells in the apical side migrate downward to the basal side and form crypt architecture (56). The results from H\&E staining of sequential sections suggested that the cells derived from the recipient luminal epithelium form the glandular epithelium by their migration and invagination in the early regeneration phase of DUM, although it is difficult to assess whether invagination of epithelial cells occurs along the lines of extracellular matrices of previous glands. It is certain that regenerated glands obtain normal function because of normal pregnancy outcomes at the sites of transplanted DUMs. Recent reports state that differentiated cells behave like stem cells and actively contribute to tissue repair upon injury, called dedifferentiation (57-59). From this point of view, differentiated luminal epithelial cells may expansively commit to tissue repair, including gland formation upon surgical damage. Therefore, "epithelial invagination" may occur in the mouse uterus and form a uterine glandular epithelium subsequent to luminal "epithelial restitution" in our mouse model.

The present study does not explain the induction mechanism of stromal and myometrial regeneration, which mainly occurs during the later periods of uterine reconstruction after day 7 . It is speculated that the stromal regeneration mechanism is different from the epithelial cells; proliferating stromal cells were scarcely detected in the cell-supply compartment at the surgical site compared with epithelial cells on day 1. In addition, stromal regeneration was not observed on day 3 , but rather on day 7 . The delay of stromal regeneration behind the epithelial regeneration might reflect the involvement of factors other than acute inflammation in stromal regeneration. Epithelial-mesenchymal transition (EMT) and the paracrine mech- 
anism from newly formed epithelium to neighboring stromal cells are the speculated inducers in stromal regeneration. EMT is a phenomenon observed in the processes of cancer progression and tissue repair (60, 61), and the paracrine mechanism, typified by Wnt signaling and Hedgehog signaling, is a key for tissue polarity determination during organogenesis (62-65). Additional assessments utilizing DMT model might provide answers for these hypotheses.

The inessentiality of ovarian hormones for uterine reconstruction in the mouse models is consistent with several physiological phenomena in humans. For example, the human uterus initiates regeneration during menstruation when the circulating estrogen level is relatively low (27-30). In addition, the human puerperal uterus can recover even after cesarean section during lactation when ovarian hormone levels are relatively low due to anovulation. The uterus may be protected by the fundamental homeostatic machinery apart from ovarian hormones, and ovarian hormones may provide appropriate uterine function beneath such nonhormonal homeostatic protection.

Clinical application of DMT could significantly alleviate patients suffering from impairment of the endometrium and myometrium through uterine regeneration. For example, uterine fibroid and adenomyosis sometimes induce myometrial and endometrial dysfunctions, with some symptoms like menorrhagia, dysmenorrhea, and infertility. Uterine surgery, such as myomectomy, adenomyomectomy, and cesarean section, increases the risk for uterine rupture in postsurgical pregnancy, perhaps due to myometrial dysfunction with poor wound healing at the surgical site. Intrauterine curettage sometimes causes intrauterine adhesion and failure of endometrial formation, which is one of the primary causes of implantation failure. To date, there are few effective therapies and preventives for these uterine dysfunctions. For such cases, transplantation of decellularized portions of human uteri obtained from other persons during surgery may help to recover uterine functions and support successful pregnancy. Importantly, DUMs from other persons will probably contribute to tissue regeneration with no immune rejection, because we can totally remove intact cells from DUMs using SDS or high hydrostatic pressure, as demonstrated by our results. Although the present DMT technology has not enabled whole uterus regeneration but has enabled partial uterine tissues to regenerate and there are limitations of this study for the translation to human uterine biology due to the relatively small graft, we believe that this technique can be a cue to the future establishment of the methods for reconstruction of whole uterus. In another aspect, the mouse DMT model can be used to unveil the molecular and cellular basis of endometrial construction. Therefore, we may apply this model to clarify the physiology of endometrial construction and the pathogenesis of the diseases in which ectopic construction of endometrium-like tissues takes place, namely endometriosis and adenomyosis, as described above. Taken together, our approach for uterine regeneration and reconstruction may produce an innovative breakthrough for future regeneration research in reproductive medicine.

\section{Methods}

Mice. WT ICR mice (Japan SLC), R26GRR mice (7), Ltf-iCre mice (66), Amhr2-Cre mice (8), Pgr-Cre mice (67), and Stat3-floxed mice (68) (Oriental Bio Service) were used in this study. R26GRR mice exhibit green emission before and red emission after Cre-mediated recombination. Ltf, Amhr2, and Pgr are expressed in the luminal epithelium, the stroma/myometrium, and all the layers of uterus, respectively. Therefore, Ltf-iCre, Amhr2-Cre, and Pgr-Cre mice have the specific expression of Cre recombinase in the luminal epithelium, the stroma/myometrium, and whole uterus, respectively. For cell lineage tracing of DMT, we generated R26GRR/Ltf-iCre and R26GRR/Amhr2-Cre mice, which have epithelium-specific and stroma/ myometrium-specific red fluorescence in the uterus. $\Delta$ Stat 3 mice were generated by crossing Stat3-floxed mice and Pgr-Cre mice. For the pregnancy experiments, recipient female mice after DMT were mated with fertile WT males, and day 1 of pregnancy was defined as the day when we recognized vaginal plug.

Preparation of DUM. Adult donor female mice (8-16 weeks old) with normal estrus cycles were sacrificed painlessly under the overdose of isoflurane. Uterine horns were excised and dissected into $5 \times 2 \mathrm{~mm}$ rectangular fragments with all the layers, including myometrium, stroma, and luminal epithelium, and briefly washed with PBS after the removal of connective and fat tissues. For the pregnancy experiments, DUMs were prepared as $10 \times 2 \mathrm{~mm}$ rectangular fragments. In the ovariectomized mouse DMT model, uterine samples were harvested from the ovariectomized mice that underwent ovariectomy 2 weeks before sacrifice and cut into $3 \times 1 \mathrm{~mm}$ rectangular fragments.

For decellularization of mouse uteri, the collected uterine fragments were treated by SDS (Wako), as we described previously (3). Briefly, the tissue fragments from donor mice were immersed in 1\% of SDS with PBS 
solution at room temperature. After SDS treatment, samples were washed with washing buffer containing $0.9 \% \mathrm{NaCl}$ (WAKO), $0.05 \mathrm{M} \mathrm{MgCl}_{2} / 6 \mathrm{H}_{2} \mathrm{O}$ (WAKO), $0.2 \mathrm{mg} / \mathrm{ml}$ DNase I (Roche Diagnostics), and 1\% Gibco penicillin-streptomycin solution (Thermo Fisher Scientific) for 1 week at $4^{\circ} \mathrm{C}$ on a shaker set at frequency of $1 \mathrm{~Hz}$ with daily buffer exchange.

$D M T$. Adult recipient female mice (8 to 16 weeks old) with normal estrus cycles were anesthetized by isoflurane and laparotomized with median abdominal incision. One of two uterine horns in each recipient mouse was partially resected at the antimesometrial side in $5 \times 2 \mathrm{~mm}$ rectangular shape, and the defect was complemented by DMT. Transplantation was performed under microscopy, and the transplanted DUMs were fixed to the recipient uteri by intermittent sutures using 10-0 nylon suture thread (Matsuda). An antiadhesive material Seprafilm (KAKEN) was put around the surgical site to prevent postsurgical adhesion. In the ovariectomized mouse DMT model, recipient mice were subjected to ovariectomy 2 weeks before transplantation and pretreated by subcutaneous injection with $100 \mathrm{ng}$ of $\mathrm{E}_{2}$ in $0.1 \mathrm{ml}$ sesame oil or vehicle 6 hours before transplantation.

Recipient mice were killed specified days after DMT, and both the surgical and the nonsurgical horns were collected from each recipient. DUMs at the surgical sites were distinguished from the original recipient uteri by the presence of suture thread. The nonsurgical horns and the surgical sites with transplanted DUMs were processed for histological analysis.

Human tissues. Human uterine tissues were obtained from women who had undergone hysterectomy due to cervical cancer. They had shown regular menstrual cycles without any hormonal treatment at least 3 months before hysterectomy. Endometrial tissues were dated according to the women's menstrual history and standard histological criteria (69).

Histology and immunohistochemistry. Formalin-fixed paraffin-embedded sections $(6 \mu \mathrm{m})$ were subjected to H\&E staining and immunohistochemistry. Antibodies to CK8 (Troma-I, Developmental Studies Hybridoma Bank, Iowa, USA), aSMA (DAKO), ER $\alpha$ (Abcam plc), PR (Abcam), Ki67 (Thermo Fisher Scientific), and pSTAT3 (Abcam, ab76315) were used. For cell counting, numbers of cells on 3 randomly selected sections were manually counted by microscopic images at $\times 40$ magnification. For the quantitative analysis of regenerating glands and stromata, 3 randomly selected sections were analyzed using Leica Laser Microdissection LMD software v7.5.1 (Leica Microsystems GmbH).

Fluorescence assay. Four percent of paraformaldehyde-fixed frozen sections (12 $\mu \mathrm{m})$ of R26GRR/ Ltf-iCre and R26GRR/Amhr2-Cre uteri were examined by fluorescence assay using a fluorescence microscope (DM5000B, Leica Microsystems GmbH).

Statistics. Statistical analyses were performed using 2-tailed Student's $t$ test. $P$ values less than 0.05 were considered statistically significant.

Study approval. All mice were housed in The University of Tokyo Animal Care Facility according to the institutional guidelines for the use of laboratory animals. The experimental procedures for mice were approved by the animal experiment committee of The University of Tokyo. The experimental procedures for humans were approved by the institutional review board of The University of Tokyo, and signed informed consent for the use of tissue was obtained from each woman.

\section{Author contributions}

$\mathrm{TH}$ and $\mathrm{YH}$ designed the study. TH and TSF performed experiments and collected the data. MM, ME, $\mathrm{LM}, \mathrm{HH}$, and KSF provided technical assistance for the experiments. TH, YH, and TSF analyzed the data. $\mathrm{SKD}, \mathrm{KSF}, \mathrm{TF}$, and YO discussed and interpreted the results. TH and YH wrote the manuscript. SKD and KSF critically reviewed the manuscript. YH supervised the study.

\section{Acknowledgments}

We thank Katie A. Gerhardt for editing the manuscript; Ken-ichi Yagami (University of Tsukuba, Tsukuba, Japan) and RIKEN BRC through the National Bio-Resource Project of MEXT, Japan, for providing R26GRR mice; Sudhansu K. Dey for providing Ltf-iCre mice; Richard Behringer (The University of Texas MD Anderson Cancer Center, Houston, Texas, USA) for providing Amhr2-Cre mice; and Francesco J. DeMayo and John P. Lydon (Baylor College of Medicine, Houston, Texas, USA for providing Pgr-Cre mice. This work was supported by an AMED grant (PRIME), Japan Society for the Promotion of Science KAKENHI grants (24689062, 15K15596, 26112703, 26112506, 16H04679, 16K15701, 15H04979, 25560191, 15K10660, 15K15597, 16H05469, 16K15700), a Cell Science Research Foundation grant, and NIH grants (HD068524 and PO1CA7783). 
Address correspondence to: Yasushi Hirota, Department of Obstetrics and Gynecology, Graduate School of Medicine, The University of Tokyo, 7-3-1 Hongo, Bunkyo-ku, Tokyo 113-8655, Japan. Phone: 81.3.3815.5411; E-mail: yhirota-tky@umin.ac.jp.

1. Revel A. Defective endometrial receptivity. Fertil Steril. 2012;97(5):1028-1032.

2. Guise JM, McDonagh MS, Osterweil P, Nygren P, Chan BK, Helfand M. Systematic review of the incidence and consequences of uterine rupture in women with previous caesarean section. BMJ. 2004;329(7456):19-25.

3. Santoso EG, et al. Application of detergents or high hydrostatic pressure as decellularization processes in uterine tissues and their subsequent effects on in vivo uterine regeneration in murine models. PLoS One. 2014;9(7):e103201.

4. Sun X, Bartos A, Whitsett JA, Dey SK. Uterine deletion of Gp130 or Stat3 shows implantation failure with increased estrogenic responses. Mol Endocrinol. 2013;27(9):1492-1501.

5. Lee JH, et al. Signal transducer and activator of transcription-3 (Stat3) plays a critical role in implantation via progesterone receptor in uterus. FASEB J. 2013;27(7):2553-2563.

6. Pawar S, Starosvetsky E, Orvis GD, Behringer RR, Bagchi IC, Bagchi MK. STAT3 regulates uterine epithelial remodeling and epithelial-stromal crosstalk during implantation. Mol Endocrinol. 2013;27(12):1996-2012.

7. Hasegawa Y, et al. Novel ROSA26 Cre-reporter knock-in C57BL/6N mice exhibiting green emission before and red emission after Cre-mediated recombination. Exp Anim. 2013;62(4):295-304.

8. Jamin SP, Arango NA, Mishina Y, Hanks MC, Behringer RR. Requirement of Bmprla for Müllerian duct regression during male sexual development. Nat Genet. 2002;32(3):408-410.

9. Haraguchi $\mathrm{H}$, et al. MicroRNA-200a locally attenuates progesterone signaling in the cervix, preventing embryo implantation. Mol Endocrinol. 2014;28(7):1108-1117.

10. Dey SK, et al. Molecular cues to implantation. Endocr Rev. 2004;25(3):341-373.

11. Cha J, Sun X, Dey SK. Mechanisms of implantation: strategies for successful pregnancy. Nat Med. 2012;18(12):1754-1767.

12. Egashira M, Hirota Y. Uterine receptivity and embryo-uterine interactions in embryo implantation: lessons from mice. Reprod Med Biol. 2013;12(4):127-132.

13. Matsumoto H, Fukui E, Yoshizawa M. Molecular and cellular events involved in the completion of blastocyst implantation. Reprod Med Biol. 2016;15(2):53-58.

14. Hou X, Tan Y, Li M, Dey SK, Das SK. Canonical Wnt signaling is critical to estrogen-mediated uterine growth. Mol Endocrinol. 2004;18(12):3035-3049.

15. Chung D, Gao F, Jegga AG, Das SK. Estrogen mediated epithelial proliferation in the uterus is directed by stromal Fgf10 and Bmp8a. Mol Cell Endocrinol. 2015;400:48-60.

16. Taniguchi K, et al. A gp130-Src-YAP module links inflammation to epithelial regeneration. Nature. 2015;519(7541):57-62

17. Gurtner GC, Werner S, Barrandon Y, Longaker MT. Wound repair and regeneration. Nature. 2008;453(7193):314-321.

18. Pickert G, et al. STAT3 links IL-22 signaling in intestinal epithelial cells to mucosal wound healing. J Exp Med. 2009;206(7):1465-1472.

19. Kida H, et al. GP130-STAT3 regulates epithelial cell migration and is required for repair of the bronchiolar epithelium. Am $J$ Pathol. 2008;172(6):1542-1554.

20. Sano S, et al. Stat3 in thymic epithelial cells is essential for postnatal maintenance of thymic architecture and thymocyte survival. Immunity. 2001;15(2):261-273.

21. Neufert C, et al. Activation of epithelial STAT3 regulates intestinal homeostasis. Cell Cycle. 2010;9(4):652-655.

22. Fang Y, Gupta V, Karra R, Holdway JE, Kikuchi K, Poss KD. Translational profiling of cardiomyocytes identifies an early Jak1/Stat3 injury response required for zebrafish heart regeneration. Proc Natl Acad Sci U S A. 2013;110(33):13416-13421.

23. Liang J, Wang D, Renaud G, Wolfsberg TG, Wilson AF, Burgess SM. The stat3/socs3a pathway is a key regulator of hair cell regeneration in zebrafish. J Neurosci. 2012;32(31):10662-10673.

24. Tadokoro T, Wang Y, Barak LS, Bai Y, Randell SH, Hogan BL. IL-6/STAT3 promotes regeneration of airway ciliated cells from basal stem cells. Proc Natl Acad Sci U S A. 2014;111(35):E3641-E3649.

25. Adamson IY, Bakowska J. Relationship of keratinocyte growth factor and hepatocyte growth factor levels in rat lung lavage fluid to epithelial cell regeneration after bleomycin. Am J Pathol. 1999;155(3):949-954.

26. Pociask DA, et al. IL-22 is essential for lung epithelial repair following influenza infection. Am J Pathol. 2013;182(4):1286-1296

27. McLennan CE, Rydell AH. Extent of endometrial shedding during normal menstruation. Obstet Gynecol. 1965;26(5):605-621.

28. Ferenczy A. Studies on the cytodynamics of human endometrial regeneration. I. Scanning electron microscopy. Am J Obstet Gynecol. 1976;124(1):64-74.

29. Ferenczy A. Studies on the cytodynamics of human endometrial regeneration. II. Transmission electron microscopy and histochemistry. Am J Obstet Gynecol. 1976;124(6):582-595.

30. Chan RW, Schwab KE, Gargett CE. Clonogenicity of human endometrial epithelial and stromal cells. Biol Reprod. 2004;70(6):1738-1750

31. Ji R, et al. The differentiation of MSCs into functional hepatocyte-like cells in a liver biomatrix scaffold and their transplantation into liver-fibrotic mice. Biomaterials. 2012;33(35):8995-9008.

32. Ott HC, et al. Perfusion-decellularized matrix: using nature's platform to engineer a bioartificial heart. Nat Med. 2008;14(2):213221.

33. Wagner DE, et al. Can stem cells be used to generate new lungs? Ex vivo lung bioengineering with decellularized whole lung scaffolds. Respirology. 2013;18(6):895-911.

34. Salvatori M, et al. Extracellular matrix scaffold technology for bioartificial pancreas engineering: state of the art and future challenges. J Diabetes Sci Technol. 2014;8(1):159-169.

35. Laronda MM, Jakus AE, Whelan KA, Wertheim JA, Shah RN, Woodruff TK. Initiation of puberty in mice following decellu- 
larized ovary transplant. Biomaterials. 2015;50:20-29.

36. Engler AJ, Sen S, Sweeney HL, Discher DE. Matrix elasticity directs stem cell lineage specification. Cell. 2006;126(4):677-689.

37. Vogel V, Sheetz M. Local force and geometry sensing regulate cell functions. Nat Rev Mol Cell Biol. 2006;7(4):265-275.

38. Swift J, et al. Nuclear lamin-A scales with tissue stiffness and enhances matrix-directed differentiation. Science. 2013;341(6149):1240104.

39. Fu J, et al. Mechanical regulation of cell function with geometrically modulated elastomeric substrates. Nat Methods. 2010;7(9):733-736.

40. Mammoto T, Ingber DE. Mechanical control of tissue and organ development. Development. 2010;137(9):1407-1420.

41. Jaalouk DE, Lammerding J. Mechanotransduction gone awry. Nat Rev Mol Cell Biol. 2009;10(1):63-73.

42. Dupont S, Morsut L, Aragona M, Enzo E, Giulitti S, Cordenonsi M, et al. Role of YAP/TAZ in mechanotransduction. Nature. 2011;474(7350):179-183.

43. Stewart CL, et al. Blastocyst implantation depends on maternal expression of leukaemia inhibitory factor. Nature. 1992;359(6390):76-79.

44. Reya T, Clevers H. Wnt signalling in stem cells and cancer. Nature. 2005;434(7035):843-850.

45. Ng A, et al. Lgr5 marks stem/progenitor cells in ovary and tubal epithelia. Nat Cell Biol. 2014;16(8):745-757.

46. Hirano T, Ishihara K, Hibi M. Roles of STAT3 in mediating the cell growth, differentiation and survival signals relayed through the IL-6 family of cytokine receptors. Oncogene. 2000;19(21):2548-2556.

47. Kishimoto T. IL-6: from its discovery to clinical applications. Int Immunol. 2010;22(5):347-352.

48. Yoshimura A, Naka T, Kubo M. SOCS proteins, cytokine signalling and immune regulation. Nat Rev Immunol. 2007;7(6):454465 .

49. Bollrath J, et al. gp130-mediated Stat3 activation in enterocytes regulates cell survival and cell-cycle progression during colitis-associated tumorigenesis. Cancer Cell. 2009;15(2):91-102.

50. Padykula HA. Regeneration in the primate uterus: the role of stem cells. Ann N Y Acad Sci. 1991;622:47-56.

51. Gargett CE, Nguyen HP, Ye L. Endometrial regeneration and endometrial stem/progenitor cells. Rev Endocr Metab Disord. 2012;13(4):235-251

52. Kaitu'u-Lino TJ, Morison NB, Salamonsen LA. Neutrophil depletion retards endometrial repair in a mouse model. Cell Tissue Res. 2007;328(1):197-206.

53. Brasted M, White CA, Kennedy TG, Salamonsen LA. Mimicking the events of menstruation in the murine uterus. Biol Reprod. 2003;69(4):1273-1280

54. Salamonsen LA, Woolley DE. Menstruation: induction by matrix metalloproteinases and inflammatory cells. J Reprod Immunol. 1999;44(1-2):1-27.

55. Maloy KJ, Powrie F. Intestinal homeostasis and its breakdown in inflammatory bowel disease. Nature. 2011;474(7351):298-306.

56. Yui S, et al. Functional engraftment of colon epithelium expanded in vitro from a single adult Lgr5 ${ }^{+}$stem cell. Nat Med. 2012;18(4):618-623.

57. Stange DE, et al. Differentiated Troy+ chief cells act as reserve stem cells to generate all lineages of the stomach epithelium. Cell. 2013;155(2):357-368.

58. Tata PR, et al. Dedifferentiation of committed epithelial cells into stem cells in vivo. Nature. 2013;503(7475):218-223.

59. Desai TJ, Krasnow MA. Stem cells: Differentiated cells in a back-up role. Nature. 2013;503(7475):204-205.

60. De Craene B, Berx G. Regulatory networks defining EMT during cancer initiation and progression. Nat Rev Cancer. 2013;13(2):97-110.

61. Lamouille S, Xu J, Derynck R. Molecular mechanisms of epithelial-mesenchymal transition. Nat Rev Mol Cell Biol. 2014;15(3):178-196.

62. Krauss S, Concordet JP, Ingham PW. A functionally conserved homolog of the Drosophila segment polarity gene hh is expressed in tissues with polarizing activity in zebrafish embryos. Cell. 1993;75(7):1431-1444.

63. Riddle RD, Johnson RL, Laufer E, Tabin C. Sonic hedgehog mediates the polarizing activity of the ZPA. Cell. 1993;75(7):14011416.

64. Gao B, et al. Wnt signaling gradients establish planar cell polarity by inducing Vangl2 phosphorylation through Ror2. Dev Cell. 2011;20(2):163-176.

65. Green JL, Inoue T, Sternberg PW. Opposing Wnt pathways orient cell polarity during organogenesis. Cell. 2008;134(4):646-656

66. Daikoku T, Ogawa Y, Terakawa J, Ogawa A, DeFalco T, Dey SK. Lactoferrin-iCre: a new mouse line to study uterine epithelial gene function. Endocrinology. 2014;155(7):2718-2724.

67. Soyal SM, et al. Cre-mediated recombination in cell lineages that express the progesterone receptor. Genesis. 2005;41(2):58-66.

68. Takeda K, Kaisho T, Yoshida N, Takeda J, Kishimoto T, Akira S. Stat3 activation is responsible for IL-6-dependent T cell proliferation through preventing apoptosis: generation and characterization of T cell-specific Stat3-deficient mice. J Immunol. 1998;161(9):4652-4660.

69. Noyes RW, Hertig AT, Rock J. Dating the endometrial biopsy. Fertil Steril. 1950;1(1):3-25. 